\title{
dspace.vutbr.cz
}

\section{Audio Dequantization Using (Co)Sparse (Non)Convex Methods}

\author{
ZÁVIŠKA, P.; RAJMIC, P.; MOKRÝ, O.
}

ICASSP 2021 - 2021 IEEE International Conference on Acoustics, Speech and Signal Processing (ICASSP)

elSBN: 978-1-7281-7605-5

DOI: https://doi.org/10.1109/ICASSP39728.2021.9414637

Accepted manuscript

(C2021 IEEE. Personal use of this material is permitted. Permission from IEEE must be obtained for all other uses, in any current or future media, including reprinting/republishing this material for advertising or promotional purposes, creating new collective works, for resale or redistribution to servers or lists, or reuse of any copyrighted component of this work in other works. ZÁVIŠKA, P.; RAJMIC, P.; MOKRÝ, O. "Audio Dequantization Using (Co)Sparse (Non)Convex Methods", ICASSP 2021 - 2021 IEEE International Conference on Acoustics, Speech and Signal Processing (ICASSP), 2021. DOI: 10.1109/ICASSP39728.2021.9414637. Final version is available at https://ieeexplore.ieee.org/document/9414637 


\title{
AUDIO DEQUANTIZATION USING (CO)SPARSE (NON)CONVEX METHODS
}

\author{
Pavel Záviška, Pavel Rajmic, Ondřej Mokrý* \\ Signal Processing Laboratory, Brno University of Technology, Brno, Czech Republic
}

\begin{abstract}
The paper deals with the hitherto neglected topic of audio dequantization. It reviews the state-of-the-art sparsity-based approaches and proposes several new methods. Convex as well as non-convex approaches are included, and all the presented formulations come in both the synthesis and analysis variants. In the experiments the methods are evaluated using the signalto-distortion ratio (SDR) and PEMO-Q, a perceptually motivated metric.
\end{abstract}

Index Terms-Audio dequantization, sparsity, cosparsity, convex, nonconvex, evaluation, perception

\section{INTRODUCTION}

The task of dequantization is to recover a signal from its quantized observation. Quantization is a nonlinear distortion that introduces perceptually unpleasant artifacts. Practically, a digital signal is always quantized, but normally the bit depth of each audio sample is so high (at least 16 bits per sample) that the effect of quantization is imperceptible. However, once the bit allocation starts to decrease, the quantization starts to be pronounced. Using a low bit depth in audio may be forced (the bandwidth limitation in communication systems [1], an inadequate setup during music recording) or intentional (compression of the signal via straightforward bit depth reduction, mild requirements on the quality). The timedomain quantization actually fits into a more complex audio coding task; recently, a novel audio coding strategy has been proposed with quantization allowed in multiple domains [2].

In the past decade, audio processing methods exploiting the sparsity of audio signals has drawn much attention. This is also true for the area of audio dequantization, but it is clear from a look into the literature that the interest in this task is much weaker than the attention paid to the closely related reconstruction problems of audio declipping [3, 4, 5] and audio inpainting (i.e., filling the missing gaps in the signal) [6, 7, 8].

The quantization limits the number of possible values the signal can attain; each original signal sample is rounded to the nearest quantization level [9]. In this study, we stick to the uniform quantizer for simplicity, although non-uniform quantizers [10] could be considered without loss of generality. In the case of the standard, so-called mid-riser uniform

\footnotetext{
*The work was supported by the project 20-29009S of the Czech Science Foundation (GAČR).
}

quantizer, all the quantization levels are equally distributed. The quantization step is $\Delta=2^{-w+1}$, with $w$ denoting the word length in bits per sample (bps). Specifically, the quantized signal $\mathrm{x}^{\mathrm{q}} \in \mathbb{R}^{N}$ is obtained according to the formula

$$
\left(\mathrm{x}^{\mathrm{q}}\right)_{n}=\operatorname{sgn}^{+}\left(\mathbf{x}_{n}\right) \cdot \Delta \cdot\left(\left\lfloor\frac{\left|\mathbf{x}_{n}\right|}{\Delta}\right\rfloor+\frac{1}{2}\right),
$$

where the $n$-th sample of the signal is indicated by the index $n$, and $\operatorname{sgn}^{+}(z)$ returns 1 for $z \geq 0$ and -1 for $z<0$.

For the sake of reviewing the state of the art, it will be convenient to discuss the issue referred to as the solution consistency. The solution to a dequantization problem is called consistent with the observed samples if each sample of the dequantized signal lies within the same quantization interval as the respective original sample does. If this is not true, the solution is called inconsistent.

Brauer et al. [1] approximate the dequantization by formulating a convex optimization problem, solved by the proximal splitting algorithms [11, 12]. They work exclusively on speech signals and no computer implementation is available, unfortunately. The method of [1] is consistent with the quantized observations.

Záviška et al. [13] follow up on the just mentioned study. They continue in exploring the potential of sparsity-based methods in reconstructing quantized audio. The authors reimplement the method of [1] but in particular, they significantly extend the range of the evaluation scenarios and introduce also the analysis (cosparse) model. They also show that using the Gabor transform in place of the discrete cosine transform used in [1] leads to improved results.

Rencker et al. [14, 15] allow inconsistency of the solution. However, the inconsistency is penalized, leading to an unavoidable existence of a user-defined parameter that balances the degrees of sparsity and inconsistency of the solution. Their formulation also leads to a convex problem, but now admitting a quick solver, which comes from the fact that the inconsistency penalty is a smooth function.

Even more recently, a deep-learning-based method has been published [16]. Nonetheless, the classical approaches have not yet been fully explored, which is why the goal of this paper is to evaluate a number of sparsity-based audio dequantization methods. In the paper, we cover the methods mentioned above but also propose several brand new approaches. 


\section{SPARSITY-BASED FORMULATION}

The above introduced consistent solution is formally characterized such that it belongs to the set $\Gamma \subset \mathbb{R}^{P}$, where

$$
\Gamma=\left\{\mathbf{x} \in \mathbb{R}^{P} \mid\left\|\mathbf{x}-\mathbf{x}^{\mathrm{q}}\right\|_{\infty}<\Delta / 2\right\} .
$$

In words, the dequantized sample cannot lie outside of the quantization interval that corresponds to the original sample. The set $\Gamma$ is a convex, multidimensional interval, or a box, which indicates the relationship to audio inpainting and declipping [2]. This observation also motivates the adaptation of efficient reconstruction algorithms to dequantization.

Given $x^{q}$, there are numerous feasible signals in $\Gamma$, making the reconstruction ill-posed. Sparsity can come into play here as a regularizer. In audio, the assumption of sparsity of the time-frequency coefficients of the signal is usually employed. Denote $\mathbf{c} \in \mathbb{C}^{Q}$ a vector of coefficients and $\mathbf{x} \in \mathbb{R}^{P}$ a time-domain signal. The regularized problem is then to find $\mathbf{x} \in \Gamma$ such that the corresponding coefficients $\mathbf{c}$ are as sparse as possible. The time-frequency transforms are assumed redundant (therefore $Q>P$ ), leading to two distinct variants based on the relationship between $\mathbf{x}$ and $\mathbf{c}$ [17]:

$$
\begin{aligned}
& \arg \min \|\mathbf{c}\|_{0} \quad \text { subject to } A^{*} \mathbf{c} \in \Gamma \text {, } \\
& \mathbf{c} \in \mathbb{C}^{Q} \\
& \underset{\mathbf{x} \in \mathbb{R}^{P}}{\arg \min }\|A \mathbf{x}\|_{0} \quad \text { subject to } \quad \mathbf{x} \in \Gamma \text {. }
\end{aligned}
$$

The symbol $\|\cdot\|_{0}$ returns the number of non-zero elements in the vector, i.e., sparsity. Since the formulation 3a uses the operator $A^{*}$ called synthesis, it is referred to as the synthesis formulation. Similarly, $3 \mathrm{~b}$ is the analysis formulation.

The condition $A^{*} \mathbf{c} \in \Gamma$ in Eq. (3a) may be equivalently written as $\mathbf{c} \in \Gamma^{*}$ with $\Gamma^{*}=\left\{\mathbf{c}^{\prime} \in \mathbb{C}^{Q} \mid A^{*} \mathbf{c}^{\prime} \in \Gamma\right\}$. The crucial observations are that both the sets $\Gamma$ and $\Gamma^{*}$ are convex and that there exist explicit formulas for the corresponding projection operators [18]. This fact is utilized by the numerical solvers described in Sec.3

Throughout the rest of the paper, we assume the use of Parseval tight frames, i.e. linear operators for which it holds $A^{*} A=$ Id [19]. Any tight frame can be simply scaled to become Parseval-tight.

\section{PROBLEMS AND ALGORITHMS}

The presence of the $\ell_{0}$ penalty makes the problems in (3) NPhard and therefore the solutions need to be approximated in practice. This section is devoted to the presentation of formulations that follow from different means of approximation, and the corresponding algorithms.

\subsection{Consistent $\ell_{1}$ minimization}

The first option is to use the $\ell_{1}$ norm instead of $\ell_{0}$ to make the whole problem convex [20, 21], allowing the use of convex optimization [22, 23].

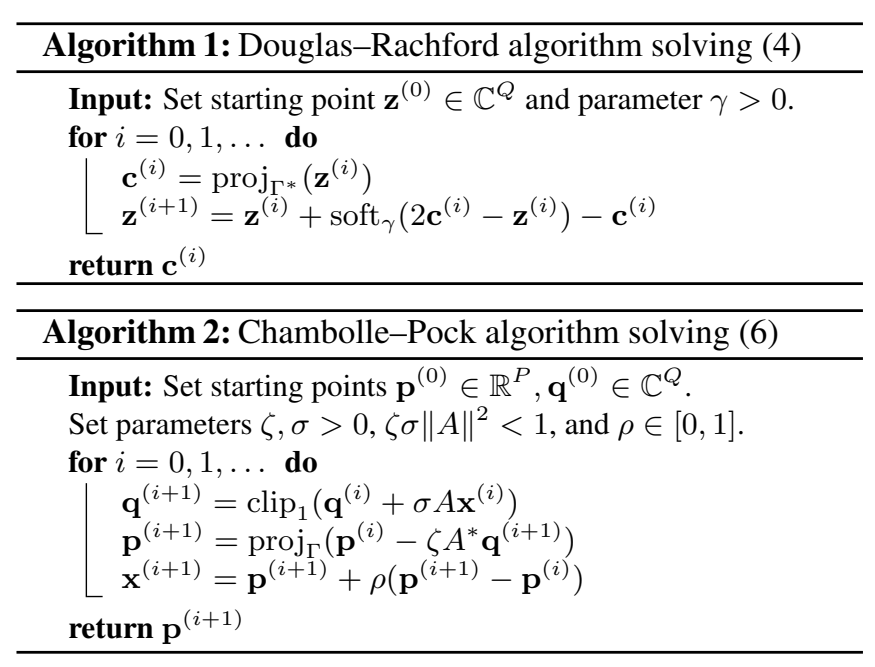

The convex relaxation of $3 \mathrm{a}$ reads

$$
\underset{\mathbf{c} \in \mathbb{C}^{Q}}{\arg \min }\|\mathbf{c}\|_{1} \quad \text { subject to } \quad A^{*} \mathbf{c} \in \Gamma .
$$

To solve (4) numerically, proximal splitting methods [11] offer a suitable choice. We show in [13] that the solution to (4) can be found via the Douglas-Rachford (DR) algorithm [11, Sec. 4]. The DR algorithm is summarized in Alg.1. Bearing in mind that $A^{*} \mathbf{c} \in \Gamma$ is equivalent to $\mathbf{c} \in \Gamma^{*}$, the DR algorithm is derived such that its first step uses the projection onto the set $\Gamma^{*}$. The projection can be computed as [18]

$$
\operatorname{proj}_{\Gamma^{*}}(\mathbf{c})=\mathbf{c}+A\left(\operatorname{proj}_{\Gamma}\left(A^{*} \mathbf{c}\right)-A^{*} \mathbf{c}\right) .
$$

The second step of the DR algorithm uses $\operatorname{soft}_{\alpha}$, the soft thresholding operator with a threshold $\alpha>0$ [20, 24].

Similar to the synthesis model, we relax $3 \mathrm{~b}$ as

$$
\underset{\mathbf{x} \in \mathbb{R}^{P}}{\arg \min }\|A \mathbf{x}\|_{1} \quad \text { subject to } \quad \mathbf{x} \in \Gamma .
$$

Compared with (4), the analysis problem (6) is more challenging because of the presence of the composite function $\|A \cdot\|_{1}$. The proximal operator of such a composition is not available even in the case of a Parseval tight frame in place of $A$ [25]. However, the Chambolle-Pock (CP) algorithm [12] can handle such an optimization problem. The CP algorithm for dequantization is summarized in Alg.2. In the algorithm, $\operatorname{clip}_{\alpha}=\mathrm{Id}-\operatorname{soft}_{\alpha}$.

\subsection{SPADQ, non-convex minimization}

Another option in approximating the problems (3) is to relax the strict relationship of $\mathbf{x}$ and $\mathbf{c}$ and approach the problem heuristically [5, 26]. The acronym SPADQ stands for the Sparse Audio Dequantizer, a natural adaptation of the Sparse Audio Declipper (SPADE) [5, 26] or Inpainter (SPAIN) [27] to the task of audio dequantization. The dequantization models are derived from a reformulation of (3) with less rigid 
coupling of the signal and the coefficients, governed by the parameter $\epsilon$. Based on [5, 26], three options are available:

$$
\begin{gathered}
\underset{\mathbf{c}, \mathbf{z} \in \mathbb{C}^{Q}}{\arg \min }\|\mathbf{z}\|_{0} \text { subject to } A^{*} \mathbf{c} \in \Gamma,\|\mathbf{c}-\mathbf{z}\|_{2} \leq \epsilon, \\
\underset{\mathbf{x} \in \mathbb{R}^{P}, \mathbf{c} \in \mathbb{C}^{Q}}{\arg \min }\|\mathbf{c}\|_{0} \text { subject to } \mathbf{x} \in \Gamma,\left\|\mathbf{x}-A^{*} \mathbf{c}\right\|_{2} \leq \epsilon, \\
\underset{\mathbf{x} \in \mathbb{R}^{P}, \mathbf{c} \in \mathbb{C}^{Q}}{\arg \min }\|\mathbf{c}\|_{0} \text { subject to } \mathbf{x} \in \Gamma,\|A \mathbf{x}-\mathbf{c}\|_{2} \leq \epsilon .
\end{gathered}
$$

The respective algorithms (S-SPADQ, S-SPADQ DR, and ASPADQ) all use an iterative routine, the principal steps of which are the adaptive hard thresholding and the projection onto $\Gamma$. Actually, the three SPADQ algorithms are identical to their counterparts for audio declipping, only the definition of the feasible set $\Gamma$ differs between SPADQ and SPADE. Due to this fact and due to a lack of space, we do not reprint the algorithms here; the reader can find them in [26].

\subsection{Inconsistent $\ell_{1}$ minimization}

The last option to approximate (3) combines the $\ell_{1}$ relaxation with the relaxation of the constraints $\mathbf{x} \in \Gamma$ or $\mathbf{c} \in \Gamma^{*}$. As a result of the constraint relaxation, the method is not consistent in the sense described above. However, it may yield a sparser solution while not being too far from the feasible set. The synthesis and analysis formulations read

$$
\begin{aligned}
& \underset{\mathbf{c} \in \mathbb{C}^{Q}}{\arg \min } \lambda\|\mathbf{c}\|_{1}+\frac{1}{2} d_{\Gamma}^{2}\left(A^{*} \mathbf{c}\right), \\
& \underset{\mathbf{x} \in \mathbb{R}^{P}}{\arg \min } \lambda\|A \mathbf{x}\|_{1}+\frac{1}{2} d_{\Gamma}^{2}(\mathbf{x}),
\end{aligned}
$$

where the symbol $d_{C}(\cdot)$ denotes the distance from the set $C$, i.e., $d_{C}(\cdot)=\left\|\cdot-\operatorname{proj}_{C}(\cdot)\right\|_{2}$, and $\lambda>0$ controls the trade-off between the sparsity and the consistency of the solution. The synthesis variant (8a) can be solved via FISTA, as shown recently in [14]. FISTA is a proximal gradient method [28, 11, 4]), accelerated thanks to the fact that $\frac{1}{2} d_{C}^{2}$ is differentiable, with the gradient $\nabla \frac{1}{2} d_{C}^{2}(\mathbf{x})=\mathbf{x}-\operatorname{proj}_{C}(\mathbf{x})$ [15]. The resulting algorithm is Alg. 3

Alternatively, we can use the DR algorithm to solve $8 \mathrm{a}$ ). From [24, Example 6.65], we know that

$$
\operatorname{prox}_{\alpha d_{C}^{2} / 2}(\mathbf{z})=\frac{1}{\alpha+1}\left(\alpha \operatorname{proj}_{C}(\mathbf{z})+\mathbf{z}\right),
$$

which is a convex combination of a point and its projection onto $C$. This formula is used as the proximal operator of the second term in $8 \mathrm{a}$. The resulting algorithm is Alg. 4 .

The analysis-based problem $8 \mathrm{~b}$ can be solved using the $\mathrm{CP}$ algorithm; see Alg.5 Note that the update of $\mathbf{p}$ uses the proximal operator presented in (9). Finally, we propose two alternatives of tackling $(8 \mathrm{~b})$ by means of another approximation. First, let us apply the DR algorithm to the problem. The proximal operator of the distance function can again be taken

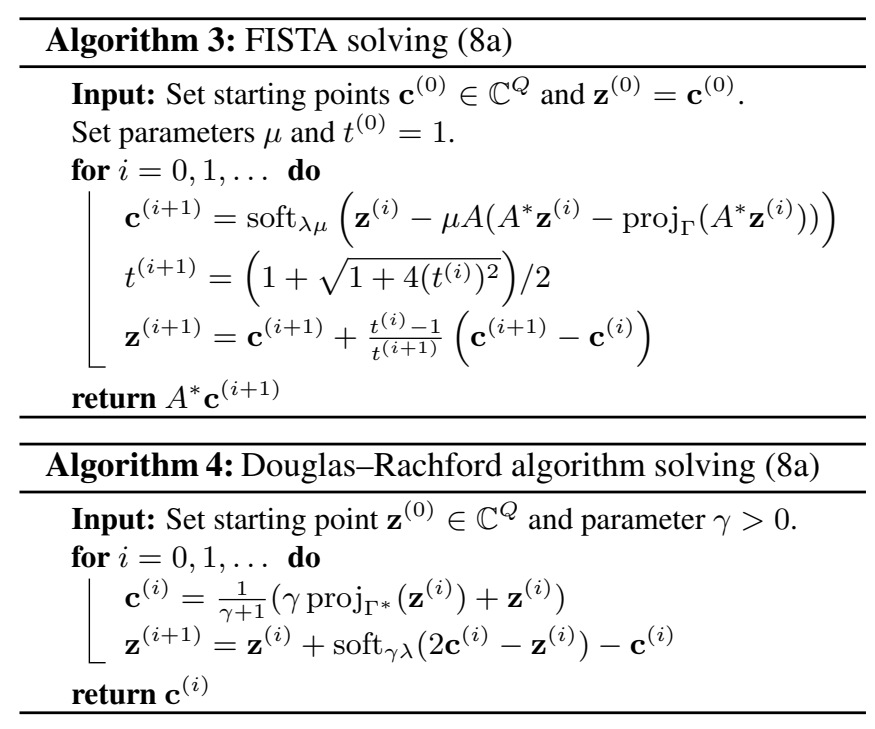

from (97. The proximal operator of $\alpha\|A \cdot\|_{1}=\alpha\|\cdot\|_{1} \circ A$ is problematic. If the involved linear operator were the synthesis, the same composition rule could be followed as in the case of the projection (5). For $A$ being the analysis, no such rule can be applied, though. Nevertheless, [25] shows that an approximation can be done using the so-called approximal operator, which turned out to be very successful in the case of audio inpainting. In our case, it takes the form

$$
\operatorname{approx}_{\alpha\|\cdot\|_{1} \circ A}(\mathbf{x})=A^{*} \operatorname{soft}_{\alpha}(A \mathbf{x}) .
$$

Substituting the proximal operator of distance in Alg. 4 with the approximal operator from (10) results in Alg.6. As the second alternative, FISTA can also be used for the approximation of the analysis variant $(8 \mathrm{~b})$, since $d_{\Gamma}$ is differentiable and the proximal operator of $\|A \cdot\|_{1}$ can be substituted with the approx, just as above. The resulting algorithm is in Alg.77.

\section{EXPERIMENTS AND RESULTS}

For the experiments, an audio database containing ten musical excerpts sampled at $44.1 \mathrm{kHz}$, with an approximate duration of 7 seconds, was used. The excerpts were extracted from the EBU SQAM database ${ }^{1}$ and thoroughly selected to cover a wide range of signal sparsity.

The signals were first peak-normalized and then quantized according to (1), using 7 different word lengths, $w=$ $2,3, \ldots, 8$. The Discrete Gabor transform (DGT) was chosen as the sparsity-promoting transform, using a Hann window 8192 samples long $(185.8 \mathrm{~ms})$. The DGT used a $75 \%$ overlap of the windows and 16384 frequency channels. The algorithms were implemented in MATLAB 2019b. They rely on the LTFAT toolbox [29] for the time-frequency operators.

The physical similarity of waveforms was measured using the $\Delta \mathrm{SDR}$, which expresses the signal-to-distortion ratio (SDR) improvement of the quantized signal to the recon-

\footnotetext{
${ }^{1}$ https://tech.ebu.ch/publications/sqamcd
} 

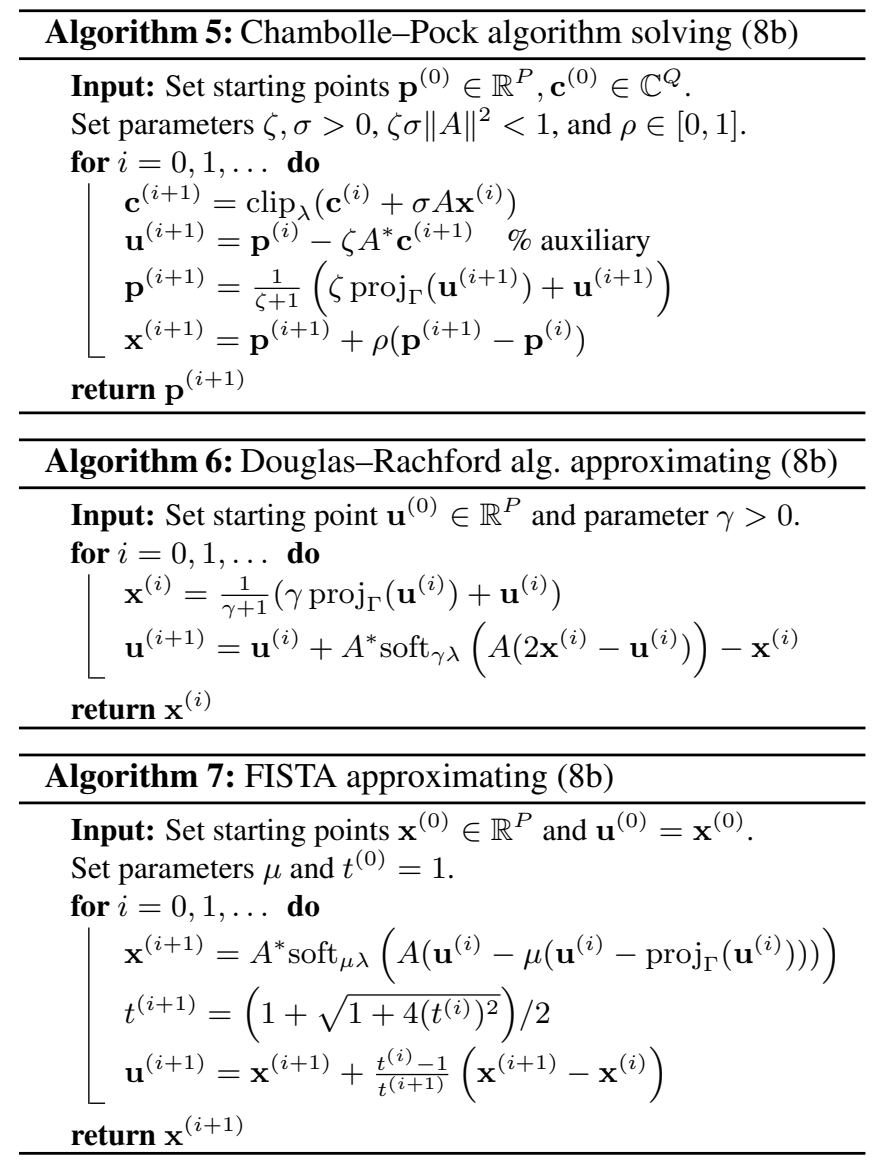

structed signal. Note that in dequantization, the SDR is equivalent to the signal-to-artifacts ratio (SAR). Since we are interested mostly in perceptual quality (which may not correspond to the SDR values), we evaluate the reconstructed signals using the PEMO-Q metric [30], which uses an objective difference grade (ODG) scale of -4 to 0 (worst to best).

The parameters of the algorithms need fine-tuning to achieve the best possible results. In the case of $\ell_{1}$ minimization, the $\triangle$ SDR values tend to gain rapidly during the first couple of iterations but then drop and stabilize at a lower value. This is explained by the fact that during the convergence, the algorithms retain the signal within the consistent area, while the $\ell_{1}$ norm of the coefficients decreases. When their $\ell_{1}$ norm is pushed too far towards zero, the waveform is also affected, tending to incorrectly settle close to the edge of the feasible quantization intervals. Interrupting the convergence at the SDR peak provides results with the most similar waveforms to the original (in practice unknown) signal. The first bar chart in Fig. 1 shows the best achievable $\triangle$ SDR values, which in our case correspond to stopping the convergence after approximately 100 iterations. Letting the algorithms fully converge yields significantly better results in terms of the perceptual metric. The PEMO-Q ODG values are presented as the second chart of Fig. 1 and they were reached after 500 iterations of each algorithm.

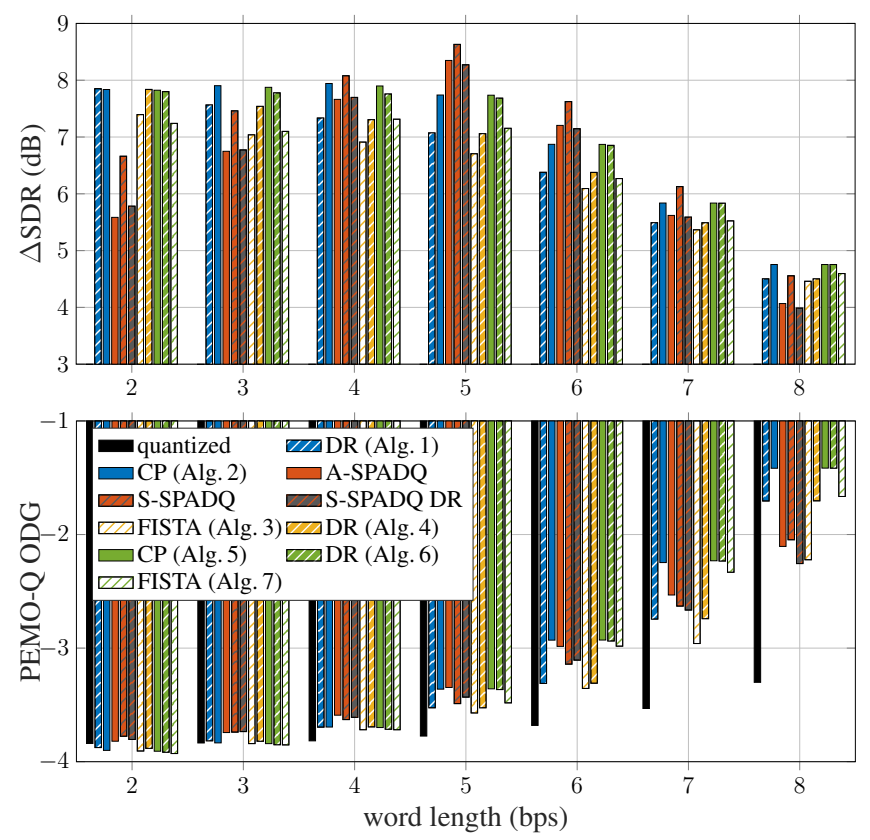

Fig. 1. Average $\Delta$ SDR and PEMO-Q ODG results.

The $\Delta$ SDR results suggest no clear winner. The SPADQ algorithms perform well for word lengths of 4-7 bps but for other tested word lengths they are outperformed by the convex methods. In the case of consistent $\ell_{1}$ minimization, it is clear that except for the 2 bps case, the analysis variant using the $\mathrm{CP}$ algorithm outperforms the synthesis variant using the DR algorithm. The results of the inconsistent problem formulations also indicate the predominance of analysis-based formulations; this behavior can also be observed in audio declipping and inpainting. The effect of inexact computation of the thresholding step (10) turns out to have negligible influence, as in the case of inpainting [25].

The PEMO-Q results indicate that for $w \geq 4 \mathrm{bps}$ all the methods improve the perceptual quality. The overall PEMO-Q results roughly correspond to the $\triangle$ SDR results. Small differences can be found in the case of SPADQ or methods based on inconsistent $\ell_{1}$ minimization. Finally, the FISTA algorithms seem to perform worse than the other methods in both the $\triangle$ SDR and PEMO-Q.

The MATLAB implementation and data are available at http://github.com/zawi01/audio_dequantization.

\section{CONCLUSION}

The paper discussed a number of sparsity-based approaches to dequantization. The audio signals were subjected to uniform quantization. The signals were reconstructed using convex and non-convex approaches, respecting strictly, or only approximately the solution consistency. None of the $\triangle$ SDR and PEMO-Q results suggest a clearly preferred method for audio dequantization. Among the convex methods, the variants involving the analysis time-frequency operator appear to give better results than the synthesis-based variants do. 


\section{REFERENCES}

[1] C. Brauer, T. Gerkmann, and D. Lorenz, "Sparse reconstruction of quantized speech signals," in 2016 IEEE ICASSP, March 2016, pp. 5940-5944.

[2] O. Mokrý, P. Rajmic, and P. Záviška, "Flexible framework for audio reconstruction," in Proceedings of the 23rd DAFx2020, Sept. 2020-21, vol. 1.

[3] P. Záviška, P. Rajmic, A. Ozerov, and L. Rencker, "A survey and an extensive evaluation of popular audio declipping methods," IEEE Journal of Selected Topics in Signal Processing, vol. 15, no. 1, pp. 5-24, 2021.

[4] K. Siedenburg, M. Kowalski, and M. Dörfler, "Audio declipping with social sparsity," in 2014 IEEE ICASSP, May 2014, pp. 1577-1581.

[5] S. Kitić, N. Bertin, and R. Gribonval, "Sparsity and cosparsity for audio declipping: a flexible non-convex approach," in 12th International Conference on Latent Variable Analysis and Signal Separation, Aug. 2015.

[6] A. Adler et al., "Audio Inpainting," IEEE Trans. Audio, Speech, Language Process., vol. 20, no. 3, pp. 922-932, 2012.

[7] O. Mokrý, and P. Rajmic, "Audio inpainting: Revisited and reweighted," IEEE/ACM Transactions on Audio, Speech, and Language Processing, vol. 28, pp. 29062918, 2020.

[8] A. Marafioti, N. Holighaus, P. Majdak, and N. Perraudin, "Audio inpainting of music by means of neural networks," in Audio Engineering Society Convention 146, Mar. 2019.

[9] U. Zölzer, DAFX: Digital Audio Effects, Wiley, 2011.

[10] Pulse code modulation (PCM) of voice frequencies, Geneva, 1988.

[11] P.L.Combettes and J.C.Pesquet, "Proximal splitting methods in signal processing," Fixed-Point Algorithms for Inverse Problems in Science and Engineering, vol. 49, pp. 185-212, 2011.

[12] A. Chambolle and T. Pock, "A first-order primal-dual algorithm for convex problems with applications to imaging," J. Math. Imaging Vis., vol. 40, no. 1, pp. 120-145, 2011.

[13] P. Záviška and P. Rajmic, "Sparse and cosparse audio dequantization using convex optimization," in 2020 43rd International Conference on Telecommunications and Signal Processing (TSP), July 2020, pp. 216-220.

[14] L. Rencker, F. Bach, W. Wang, and M.D. Plumbley "Fast iterative shrinkage for signal declipping and dequantization," in iTWIST'18, 2018.

[15] L. Rencker, F. Bach, W. Wang, and M.D. Plumbley, "Sparse recovery and dictionary learning from nonlinear compressive measurements," IEEE Trans. Signal Process., vol. 67, no. 21, pp. 5659-5670, 2019.
[16] C. Brauer, Z. Zhao, D. Lorenz, and T. Fingscheidt, "Learning to dequantize speech signals by primal-dual networks: an approach for acoustic sensor networks," in 2019 IEEE ICASSP, May 2019, pp. 7000-7004.

[17] S. Nam, M. Davies, M. Elad, and R. Gribonval, "The cosparse analysis model and algorithms," Appl. Comput. Harmon. Anal., vol. 34, no. 1, pp. 30-56, 2013.

[18] P. Rajmic, P. Záviška, V. Veselý, and O. Mokrý, “A new generalized projection and its application to acceleration of audio declipping," Axioms, vol. 8, no. 3, 2019.

[19] O. Christensen, Frames and Bases, An Introductory Course, Birkhäuser, Boston, 2008.

[20] D. L. Donoho and M. Elad, "Optimally sparse representation in general (nonorthogonal) dictionaries via $\ell_{1}$ minimization," Proceedings of The National Academy of Sciences, vol. 100, no. 5, pp. 2197-2202, 2003.

[21] M. Fornasier, Theoretical Foundations and Numerical Methods for Sparse Recovery, De Gruyter, 2010.

[22] S. S. Chen, D. L. Donoho, and M. A. Saunders, "Atomic decomposition by basis pursuit," SIAM Review, vol. 43, No. 1, pp. 129-159, 2001.

[23] S. P. Boyd and L. Vandenberghe, Convex Optimization, Cambridge University Press, 2004.

[24] A. Beck, First-Order Methods in Optimization, SIAMSociety for Industrial and Applied Mathematics, 2017.

[25] O. Mokrý and P. Rajmic, "Approximal operator with application to audio inpainting," Signal Processing, vol. 179, 2021.

[26] P. Záviška, P. Rajmic, O. Mokrý, and Z. Průša, "A proper version of synthesis-based sparse audio declipper," in 2019 IEEE ICASSP, May 2019, pp. 591595.

[27] O. Mokrý, P. Záviška, P. Rajmic, and V. Veselý, "Introducing SPAIN (SParse Audio INpainter)," in 2019 27th European Signal Processing Conference (EUSIPCO). Sept. 2019.

[28] I. Daubechies, M. Defrise, and C. De Mol, "An iterative thresholding algorithm for linear inverse problems with a sparsity constraint," Commun. Pure Appl. Math, vol. 57, no. 11, pp. 1413-1457, 2004.

[29] Z. Průša et al., "The Large Time-Frequency Analysis Toolbox 2.0," in Sound, Music, and Motion, pp. 419442. Springer International Publishing, 2014.

[30] R. Huber and B. Kollmeier, "PEMO-Q-A new method for objective audio quality assessment using a model of auditory perception," IEEE Trans. Audio Speech Language Proc., vol. 14, no. 6, pp. 1902-1911, 2006. 